\title{
Impact of Mucin1 knockdown on the phenotypic characteristics of the human hepatocellular carcinoma cell line SMMC-7721
}

\author{
QIONGSHU LI ${ }^{1 *}$, FENGLI WANG ${ }^{1,2^{*}}$, GUOMU LIU $^{1}$, HONGYAN YUAN $^{1}$, TANXIU CHEN $^{1}$, JUAN WANG $^{1}$, \\ FEI XIE ${ }^{1}$, RUIPING ZHAI ${ }^{1}$, FANG WANG ${ }^{1}$, YINGYING GUO ${ }^{1}$, WEIHUA NI ${ }^{1}$ and GUIXIANG TAI ${ }^{1}$ \\ ${ }^{1}$ Department of Immunology, College of Basic Medical Science, Jilin University, Changchun, Jilin 130021; \\ ${ }^{2}$ Dalian Municipal Central Hospital, Dalian, Liaoning 116033, P.R. China
}

Received February 13, 2014; Accepted March 26, 2014

DOI: $10.3892 /$ or.2014.3136

\begin{abstract}
Mucin1 (MUC1) is a transmembrane glycoprotein that plays a key role as an oncogene in the tumorigenesis of many human adenocarcinomas. However, the role of MUC1 in human hepatocellular carcinoma (HCC) progression remains unclear. In the present study, we silenced MUC1 to investigate its effect on the human HCC cell line SMMC-7721 and found that knockdown of MUC1 significantly inhibited cell proliferation, enhanced cell-cell aggregation and induced apoptosis. No significant differences were found in in vitro migration or invasion. We also observed that knockdown of MUC1 decreased the translocation of $\beta$-catenin to the nucleus, reduced the activity of $\mathrm{T}$ cell factor and blocked the expression of cyclin D1 and c-Myc. In addition, MUC1 knockdown enhanced the expression of E-cadherin, a molecular chaperone of $\beta$-catenin that plays an important role in cell-cell aggregation. In vivo assays demonstrated that there was no tumor growth in mice injected with MUC1-silenced cells. Global gene expression analysis showed that a series of genes encoding molecules in the $\mathrm{Wnt} / \beta$-catenin, nuclear factor- $\kappa \mathrm{B}(\mathrm{NF}-\kappa \mathrm{B})$, mitogen-activated protein kinase (MAPK), insulin, transforming growth factor $\beta$ (TGF- $\beta$ ) and vascular endothelial growth factor (VEGF) signaling pathways were all influenced by the knockdown of MUC1, and these may contribute to the phenotypic alterations observed. Collectively, our results indicate that MUC1 plays a key role in $\mathrm{HCC}$ tumorigenesis.
\end{abstract}

\section{Introduction}

Mucin1 (MUC1), a transmembrane glycoprotein of the mucin family, is expressed on the apical surface of most glandular

Correspondence to: Professor Guixiang Tai, Department of Immunology, College of Basic Medical Science, Jilin University, 126 Xinmin Street, Changchun, Jilin 130021, P.R. China

E-mail: taiguixiang@163.com

${ }^{*}$ Contributed equally

Key words: Mucin1, gene silencing, hepatocellular carcinoma, growth inhibition, apoptosis, aggregation epithelia in normal tissue and is overexpressed in most adenocarcinomas with aberrant glycosylation and loss of apical expression (1-5). MUC1 consists of a large extracellular $\mathrm{N}$-terminal subunit containing a variable number of tandem repeats (VNTRs) region and a C-terminal subunit that resides on the cell surface as a heterodimeric complex via a strong noncovalent interaction. The C-terminal subunit is composed of a 58-amino acid extracellular domain, a 28-amino acid transmembrane domain (TM) and a 72-amino acid cytoplasmic tail (CT) (6-8). MUC1-CT is highly conserved between different species and contains 7 tyrosine residues that can be phosphorylated by multiple kinases $(9,10)$.

Previous studies have reported that aberrantly overexpressed MUC1 influences cell adhesion(11), motility, migration, metastasis (12-14) and cell-cell aggregation (15). Studies have shown that MUC1-CT is involved in many signaling pathways, including Wnt/ $\beta$-catenin (16), c-Src (17), Grb2/Sos (18), p53 $(19,20), \operatorname{GSK} 3 \beta(16), \operatorname{EGFR}(21,22)$ and NF- $\mathrm{BB}(23,24)$, that regulate the processes of cell survival, proliferation and apoptosis. $\beta$-catenin is a major effector of the Wnt signaling pathway, and it interacts with MUC1-CT at an SXXXXXSSLS site. This interaction blocks the GSK3 $\beta$-induced degradation of $\beta$-catenin and promotes the translocation of $\beta$-catenin to the nucleus. In the nucleus, $\beta$-catenin forms a complex with the transcription factors lymphoid enhancer factor/T cell factor (LEF/TCF) and activates the transcription of Wnt-responsive genes such as cyclin D1 and c-Myc to regulate cell proliferation $(25,26)$. Further studies have demonstrated that MUC1 plays a key role as an oncogene in the formation and progression of lung (27), breast (28), ovarian (29) pancreatic and gastric carcinomas $(30,31)$.

Several reports, including our studies, have shown that MUC1 is also expressed in hepatocellular carcinoma (HCC) cell lines, such as SNU-475, SNU-449, Mahlavu (32) and SMMC-7721, and in liver carcinoma, cirrhotic liver, and normal liver tissues (33). However, the role of MUC1 in HCC progression remains unclear. In this study, we analyzed whether MUC1 plays a crucial role in HCC progression.

\section{Materials and methods}

Cell culture. The HCC cell line SMMC-7721 was purchased from the Cell Bank of the Shanghai Institute of Cell Biology, 
Chinese Academy of Sciences. Cells were cultured in Iscove's modified Dulbecco's medium (IMDM) supplemented with $100 \mathrm{U} / \mathrm{ml}$ penicillin, $100 \mu \mathrm{g} / \mathrm{ml}$ streptomycin, and $10 \%$ fetal bovine serum (FBS; Gibco-BRL, Carlsbad, CA, USA) in an incubator at $37^{\circ} \mathrm{C}$ and $5 \% \mathrm{CO}_{2}$. The stable MUC1-knockdown cells and negative control cells were maintained with $600 \mu \mathrm{g} / \mathrm{ml}$ G418 (Sigma).

Generation of stable MUC1 knockdown in SMMC-7721 cells. Small interfering RNA (siRNA) oligonucleotides were synthesized to target sequences of the MUC1 gene: GACTGATGCCAGTAGCACT, GenBank accession No. J05582. The siRNA was inserted into the expression vector pGCsilencer ${ }^{\mathrm{TM}}$ U6.Neo.GFP (Genechem Co., Ltd., Shanghai, China). A nonspecific siRNA was used as a negative control. The siRNA expression plasmids were transfected into SMMC-7721 cells using Lipofectamine 2000 (Invitrogen). Cells were screened with $1,200 \mu \mathrm{g} / \mathrm{ml} \mathrm{G} 418$ for 3 weeks. Three independent MUC1-knockdown clones (MR1-C6, MR1-D4 and MR1-D9) and a negative control clone (NC) were isolated and identified by reverse transcription-polymerase chain reaction (RT-PCR) and western blotting.

$R T-P C R$ and quantitative real-time PCR (qRT-PCR). Cells were harvested, RNA was isolated using TRIzol (Invitrogen Life Technologies). Total RNA was converted to cDNA using M-MLV reverse transcriptase and oligo (dT) primers (Promega Corporation, Madison, WI, USA) according to the manufacturer's protocol. Reverse transcribed products were used to amplify MUC1 by RT-PCR using Ex Taq DNA polymerase (Takara Bio, Inc., Shiga, Japan). $\beta$-actin was used as an internal control gene. Primers sequences used for RT-PCR were: 5'-GGTCTTGCTGGTCTTAGGAGAGAC-3' (forward) and 5'-CTGAAGTCCAGCTGACCCTGTAGCTTCACG-3 (reverse) for MUC1, and 5'-GTTGCTATCCAGGCTGTGC-3' (forward) and 5'-AGCACTGTGTTGGCGTAAG-3' (reverse) for $\beta$-actin. The length of RT-PCR was 30 cycles. Amplification products were separated by $1.5 \%$ agarose gel, and DNA was visualized by a Gel Image System (Tanon). qRT-PCR was performed using a FastStart Universal SYBR-Green Master (Rox; Roche) on an ABI7300 instrument. The primer sequences used for qRT-PCR were: 5'-GCGTTTCCCAGAGTCATC-3' (forward) and 5'-CCTCCCTTCAACACTTCCT-3' (reverse) for cyclin D1, and 5'-TACATCCTGTCCGTCCAA-3' (forward) and 5'-TTTCCTTACGCACAAGAGTT-3' (reverse) for c-Myc, and 5'-AGTTGCGTTACACCCTTTC-3' (forward) and 5'-CCTTCACCGTTCCAGTTT-3' (reverse) for $\beta$-actin. The expression of each investigated gene was normalized to the housekeeping gene $\beta$-actin. Data were calculated using the $2^{-\Delta \Delta C T}$ method (34) and are presented as the fold change in gene expression relative to the negative control sample. For the negative control sample, $2^{-\Delta \Delta \mathrm{CT}}=1$.

Cell viability assay. Cell viability was determined at different time points using a WST-1 cell viability assay according to the manufacturer's protocol (Roche Diagnostics, Mannheim, Germany). Triplicate wells containing $5 \times 10^{3}$ cells were evaluated for viability. The absorbance was measured using a microplate reader at a wavelength of $450 \mathrm{~nm}$ (BioTek Instruments, Inc., Winooski, VT, USA). The relative cell viability was calculated as the A450 nm (MUC1-silenced clones at $\mathrm{Tn}) / \mathrm{A} 450 \mathrm{~nm}$ (NC at Tn) x 100\%.

Colony formation assay. Cells $\left(2 \times 10^{3}\right)$ were seeded into 6 well-plates for 3 weeks. Cell clones were stained with crystal violet staining solution (Sigma) and the colony size and number were observed. Colony numbers that contained $>10$ cells were counted and analyzed, the colony formation ratio was calculated as (colony number / seeded cell number) $\mathrm{x} 100 \%$. Data are expressed as a ratio of the results obtained with each MUC1-knockdown clone and the negative control.

Cell cycle analysis. Cells $\left(1 \times 10^{6}\right)$ were harvested, washed with PBS, fixed with $70 \%$ ice-cold ethanol for $30 \mathrm{~min}$ and washed with PBS. Subsequently, cells were incubated in staining buffer with $\mathrm{PI} / \mathrm{RNase}$ (BD Biosciences) for $30 \mathrm{~min}$ at $4^{\circ} \mathrm{C}$ in the dark. The cell cycle was analyzed by flow cytometry (FACSCalibur; BD Biosciences).

Assays for cell migration and invasion. For migration assay, the scratch test was performed (35). Matrigel invasion by cells was studied using transwell chambers with $8-\mu \mathrm{m}$ pore size filters (Corning Incorporated, Corning, NY, USA) coated with Matrigel matrix (BD Biosciences) in a 24-well plate (36).

Cell-cell aggregation assay. Constant stirring method was performed to evaluate the cell-cell aggregation (37). Briefly, cells $\left(2.5 \times 10^{5}\right)$ were seeded into 24 -well plates. Plates were incubated at $37^{\circ} \mathrm{C}$ with constant stirring $(150 \mathrm{rpm})$ for $2 \mathrm{~h}$. Cells were then fixed with glutaraldehyde at time zero and at the end of the incubation. Aggregates were photographed under a microscope, and isolated cells were counted. The cell-cell aggregation index $=1$ - (number of isolated cells at $2 \mathrm{~h} /$ number of isolated cells at $0 \mathrm{~h}$ ) and was normalized to the data obtained with the negative control.

Annexin V-phycoerythrin staining analysis. Cells $\left(1 \times 10^{5}\right)$ were grown on slides in triplicate in 24-well plates and cultured for $48 \mathrm{~h}$. Then, cells were washed with PBS and incubated in $500 \mu \mathrm{l}$ binding buffer with $1 \mu \mathrm{l}$ Annexin V-PE (BD Biosciences) for $15 \mathrm{~min}$ in the dark at room temperature. The slides were coverslipped with glycerol and then examined and imaged on an inverted fluorescence microscope (IX71; Olympus). Annexin V-positive cell rate was calculated as (Annexin V-positive cell number / total cell number) x 100\%.

Western blot analysis. Western blotting was performed as previously described (36). Briefly, cells were lysed with RIPA lysis buffer, and the protein concentrations in cell lysates were measured using a BCA protein assay Kit (Beyotime Biotechnology, Jiangsu, China). Nuclear and cytoplasmic protein extracts were isolated using a cytoplasmic and nuclear protein extraction kit (Thermo Scientific) according to the manufacturer's protocol. Equal amounts of cell lysate protein were separated by $10 \%$ SDS-PAGE and transferred to PVDF membranes (Millipore, Billerica, MA, USA). The primary antibodies used were antibodies against MUC1 (GP1.4) (1:2,000; NeoMarkers), c-Myc (1:1,000), cyclin D1 (1:1,000), $\beta$-actin $(1: 2,000), \mathrm{I \kappa B} \alpha(1: 2,000)$ and Lamin B1 $(1: 2,000$; all 

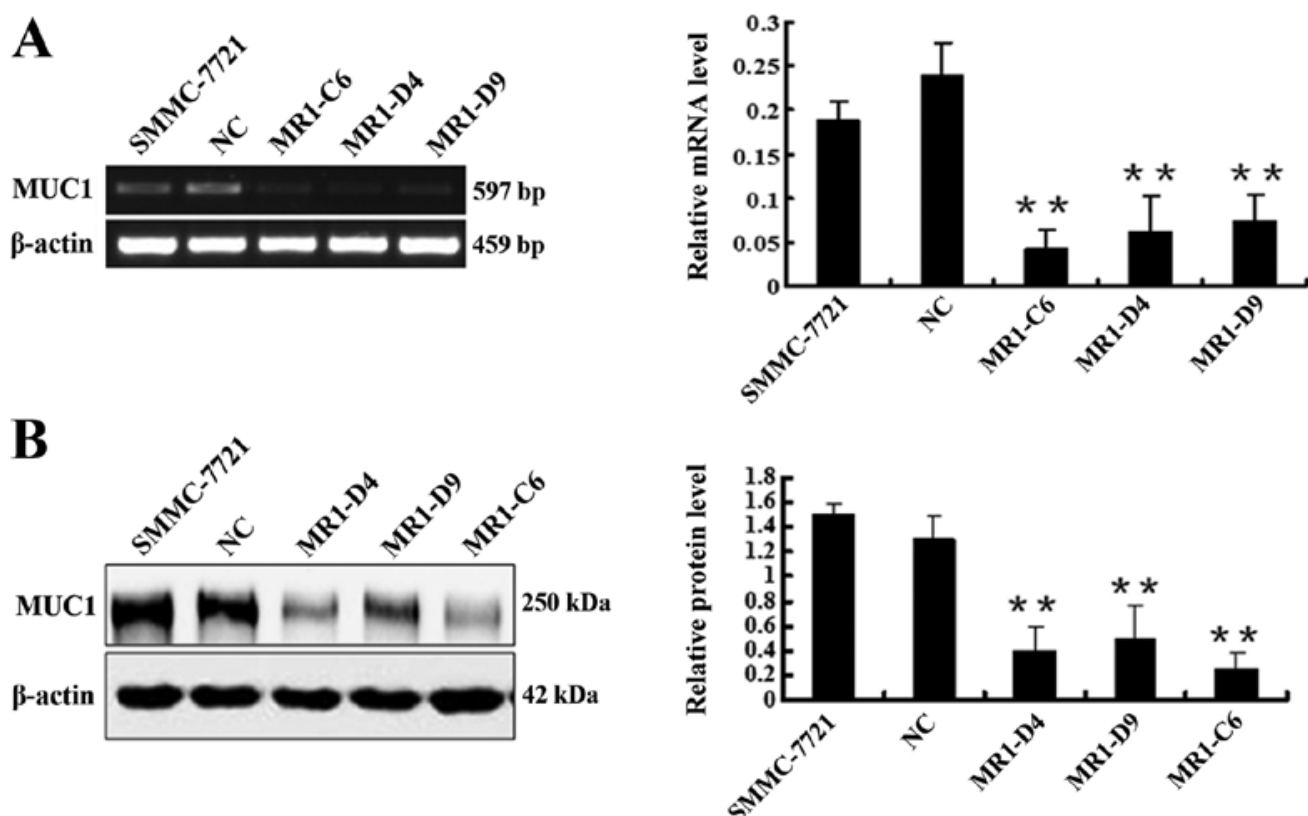

Figure 1. Knockdown of MUC1 by siRNA in SMMC-7721 cells. (A) MUC1 mRNA expression was detected by reverse transcription-polymerase chain reaction (RT-PCR) in SMMC-7721 cells and clones NC, MR1-C6, MR1-D4 and MR1-D9 using $\beta$-actin as an internal control reference gene. The bar charts represent relative mRNA levels calculated from the relative band density ratio of MUC1/ $\beta$-actin. (B) Western blotting for MUC1 expression in SMMC-7721 cells and clones NC, MR1-C6, MR1-D4 and MR1-D9 after normalization to $\beta$-actin. Bars represent the relative protein level calculated by the ratio of MUC1/ $\beta$-actin. Densitometric scanning of band intensities was obtained from three separate experiments to quantify the change in protein expression. Data are expressed as the means \pm SDs of 3 independent experiments. ${ }^{* *} \mathrm{P}<0.01$ compared with the NC.

from Epitomics, Burlingame, CA, USA), $\beta$-catenin (1:1,000; BD Biosciences), E-cadherin (Proteintech), caspase-3 (Santa Cruz Biotechnology).

Co-immunoprecipitation analysis. Cell lysates were first precleared with protein $\mathrm{G}$ agarose beads (Promega) for $3 \mathrm{~h}$ at $4^{\circ} \mathrm{C}$ and, subsequently, equal amounts of sample lysate were incubated with either $1.0 \mu \mathrm{g}$ of mouse IgG or anti-MUC1-CT antibody (Ab-5; Neomarker) for $16 \mathrm{~h}$ at $4^{\circ} \mathrm{C}$, followed by precipitation with protein $\mathrm{G}$ agarose beads. Immunoprecipitated proteins from cell lysates and total cell lysates were subjected to immunoblot analysis with anti- $\beta$-catenin.

Luciferase reporter assay. Cells were transfected using Lipofectamine 2000 (Invitrogen) according to the manufacturer's protocol with $1.0 \mu \mathrm{g} /$ well of TOPflash and FOPflash plasmids (Upstate Biotechnology, Inc., Lake Placid, NY, USA) plus $0.05 \mu \mathrm{g} /$ well of phRL-TK (Promega) to normalize transfection efficiency (36).

In vivo tumor growth assays and immunohistochemical staining. BALB/c nude mice (4-6 weeks old) were purchased from Beijing HFK Bioscience Co., Ltd., China. Animals were maintained in specific pathogen-free conditions and environment under controlled conditions of light and humidity. Animal experiments were carried out in accordance with the National Institutes of Health Guide for the Care and Use of Laboratory Animals. Mice were randomly divided into 4 groups (5 animals/group), designated the SMMC-7721 group, the NC group, the MR1-C6 group and the MR1-D4 group. Cells $\left(2 \times 10^{6}\right)$ were subcutaneously injected into the right flank of each mouse. On day 35 post-injection, the tumors were dissected, fixed in $10 \%$ neutral-buffered formalin and embedded in paraffin for immunohistochemical staining using primary antibody mouse anti-MUC1 monoclonal antibody (GP1.4) and an UltraSensitiveTM SP (Mouse/Rabbit) IHC Kit (MaiXin.BIO., Fuzhou, China).

Global gene expression analysis by microarray. NC, MR1-C6 and MR1-D4 cells were harvested, and total RNA was isolated using TRIzol (Invitrogen Life Technologies) as described above. Global gene expression analysis was performed using Roche NimbleGen microarrays (Kang Chen Bio-tech, Shanghai, China).

Statistical analysis. All statistical analyses were performed using unpaired Student's t-tests, and $\mathrm{P}<0.05$ was considered to indicate a statistically significant result.

\section{Results}

Establishment of stable MUC1 knockdown in SMMC-7721 cells. To determine the effect of MUC1 expression in HCC, we silenced MUC1 in SMMC-7721 cells using RNAi. SMMC-7721 cells were transfected with MUC1-targeted or nonspecific siRNA in the expression vector pGCsilencer ${ }^{\mathrm{TM}} \mathrm{U} 6 . \mathrm{Neo}$. GFP. We identified three independent SMMC-7721-MUC1knockdown stable cell clones (termed MR1-C6, MR1-D4 and MR1-D9) and a negative control clone termed NC by RT-PCR (Fig. 1A) and western blotting (Fig. 1B). These results showed that MUC1 expression in the MR1-C6, MR1-D4 and MR1-D9 cell clones was significantly decreased when compared to $\mathrm{NC}$ or SMMC-7721 cells $(\mathrm{P}<0.01)$. The silencing efficiency in clones MR1-C6 and MR1-D4 reached 82.34 and $74.53 \%$, 

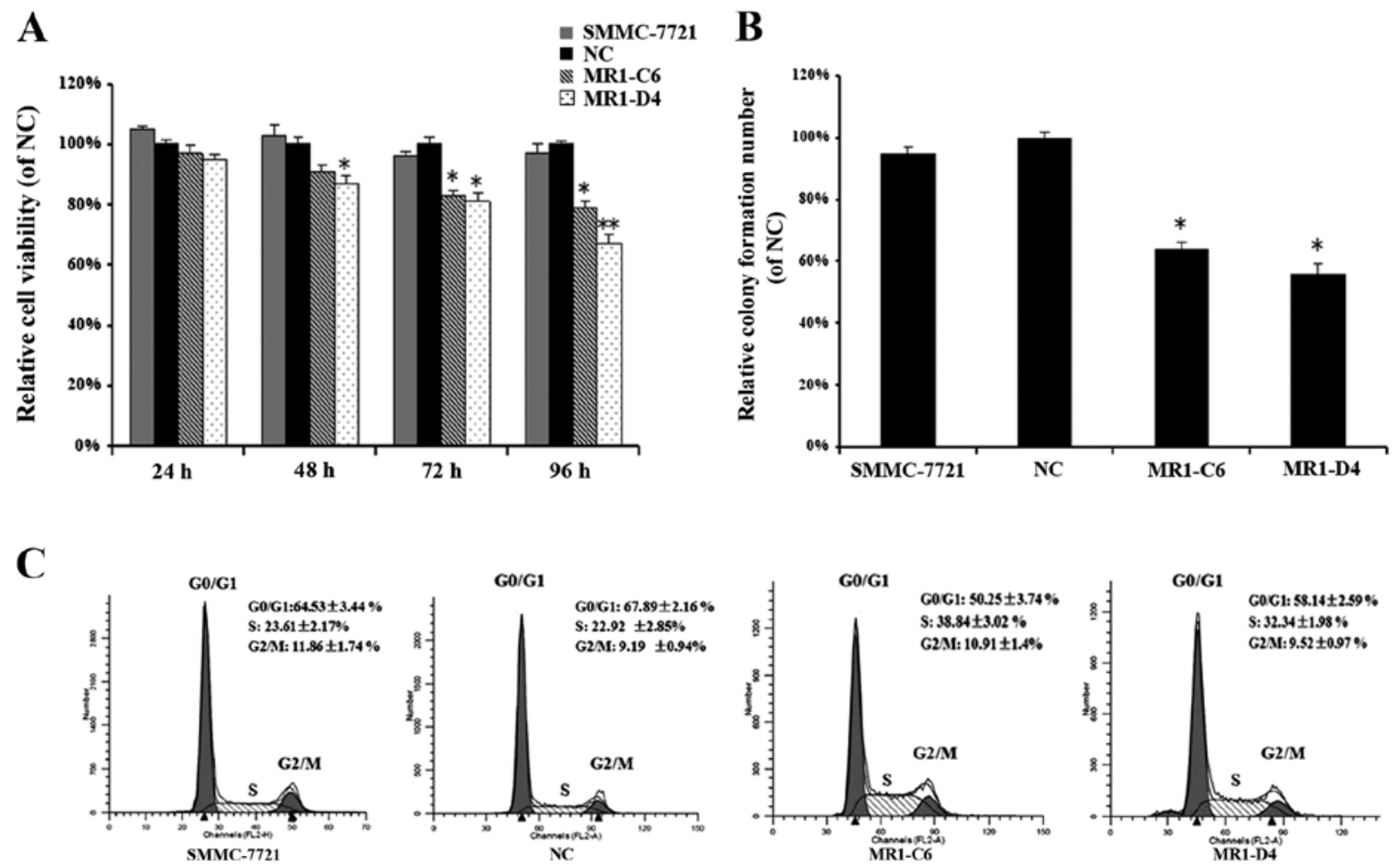

Figure 2. Knockdown of MUC1 inhibits cell proliferation and arrests cells in the S-phase. (A) Cell viability was determined by the WST-1 assay. (B) For the colony formation assay, the colony size and number were observed. Colony numbers that contained $>10$ cells were counted and analyzed; the colony formation ratio was calculated as (colony number / seeded cell number) x100\%. Data are expressed as a ratio of the results obtained with each MUC1-knockdown clone and the negative control (NC) from three independent experiments. "P<0.05 compared with NC. (C) Cell cycle analysis. The distribution of cells in G0/G1, S and $\mathrm{G} 2 / \mathrm{M}$ phases was analyzed by flow cytometry.

respectively. Therefore, we selected the MR1-C6 and MR1-D4 clones for subsequent studies.

Knockdown of MUC1 expression suppresses SMMC-7721 cell proliferation and arrests cells in S-phase. To investigate the influence of MUC1 knockdown on SMMC-7721 cell proliferation, we performed WST-1 cell viability and colony formation assays. WST-1 assays showed that the viability of MR1-C6 and MR1-D4 clones was significantly reduced in a time-dependent manner when compared to NC or SMMC-7721 cells (Fig. 2A) $(\mathrm{P}<0.05)$. Colony numbers were decreased in MR1-C6 and MR1-D4 cells when compared to NC or SMMC-7721 cells (Fig. 2B) $(\mathrm{P}<0.05)$. We also analyzed the cell cycle in MR1-C6, MR1-D4, SMMC-7721 and NC cells using flow cytometry. These results showed that MR1-C6 and MR1-D4 cells had a higher percentage of cells in the S-phase and fewer in the G0/G1 phase when compared to NC or SMMC-7721 cells (Fig. 2C), demonstrating that knockdown of MUC1 in SMMC-7721 cells inhibits cell proliferation and induces cell cycle arrest in the $\mathrm{S}$-phase.

Knockdown of MUC1 expression alters the $\beta$-catenin signaling pathway by blocking $\beta$-catenin translocation to the nucleus. Numerous reports have shown that MUC1 is involved in the $\beta$-catenin signaling pathway by binding to $\beta$-catenin. Therefore, we investigated the interaction between MUC1 and $\beta$-catenin in SMMC-7721 cells by co-immunoprecipitation. As shown in Fig. 3A, $\beta$-catenin was detected and was directly bound to MUC1-CT in SMMC-7721 cells. Furthermore, knockdown of MUC1 expression decreased the efficiency of the interaction between MUC1-CT and $\beta$-catenin. To evaluate the effect of MUC1 gene silencing on $\beta$-catenin subcellular localization, we used western blotting and showed that cytoplasmic $\beta$-catenin levels were significantly increased (Fig. 3B) $(\mathrm{P}<0.05)$, while nuclear $\beta$-catenin levels were significantly decreased (Fig. 3C) $(\mathrm{P}<0.05)$ in MR1-C6 and MR1-D4 cells when compared to NC or SMMC-7721 cells. These data indicate that knockdown of MUC1 blocks the translocation of $\beta$-catenin from the cytoplasm to the nucleus.

As the Wnt pathway is known to be involved in tumor cell proliferation, we performed a luciferase reporter assay to determine the effect of the MUC1-CT/ $\beta$-catenin interaction on the activation of Wnt signaling in SMMC-7721 cells. The results showed that TOPflash/FOPflash reporter activity in MR1-C6 and MR1-D4 cells was significantly lower than the activity in NC cells (Fig. 3D) $(\mathrm{P}<0.05)$. We used qRT-PCR and western blotting to evaluate the expression of cyclin D1 and c-Myc, which have been confirmed as target genes of the $\beta$-catenin signaling pathway that stimulate cell proliferation. As shown in Fig. 3E and F, knockdown of MUC1 significantly reduced both the mRNA and protein levels of cyclin D1 and c-Myc $(\mathrm{P}<0.05)$. The above data suggest that MUC1 gene silencing inhibits SMMC-7721 proliferation by ablating the $\beta$-catenin signaling.

Knockdown of MUC1 expression enhances cell-cell aggregation. We examined the effect of MUC1 knockdown on the cell-cell aggregation, migration and invasion of SMMC-7721 
A

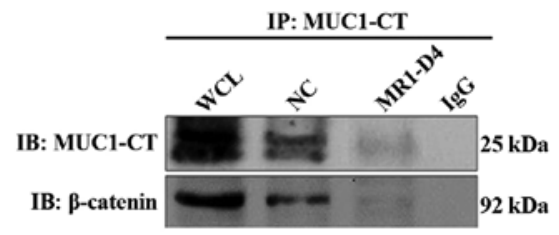

B

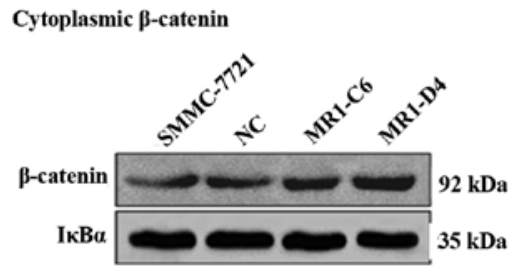

C

Nuclear $\beta$-catenin

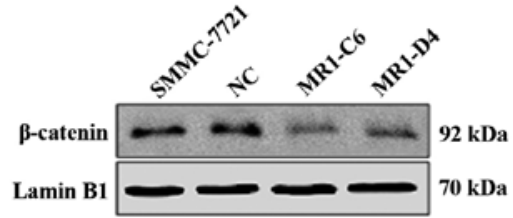

D

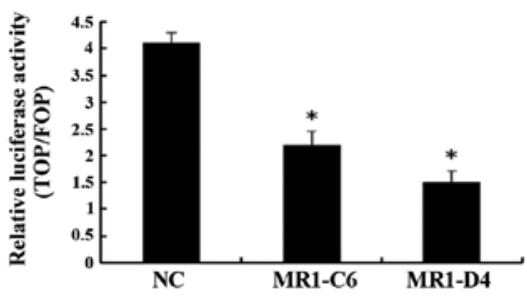

E
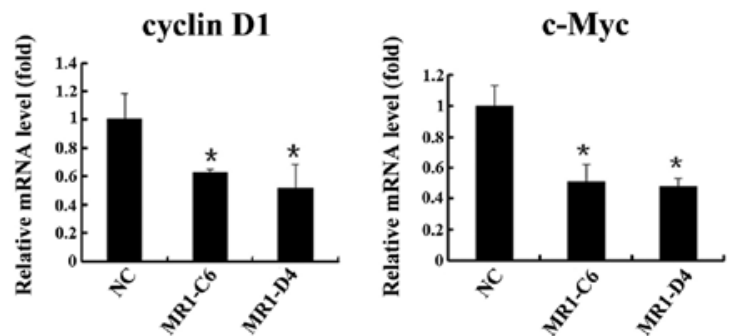

F

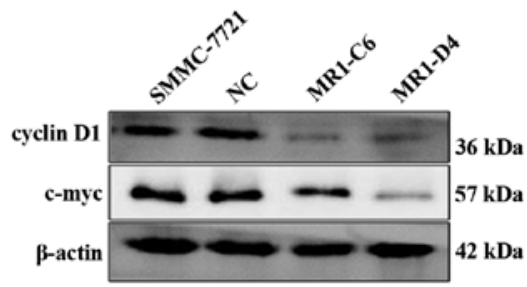

Figure 3. Knockdown of MUC1 expression alters the $\beta$-catenin signaling pathway by blocking $\beta$-catenin translocation to the nucleus. (A) Cell lysates from NC and MR1-D4 clones were subjected to immunoprecipitation (IP) with anti-MUC1-CT antibody or normal IgG and then immunoblotted (IB) with anti- $\beta$-catenin antibody. Whole cell lysate (WCL) was not subjected to immunoprecipitation. (B) Cytoplasmic extracts from SMMC-7721, NC, MR1-C6 and MR1-D4 cells were analyzed by western blotting for the expression of $\beta$-catenin. Cytoplasmic I $\mathrm{B} \alpha$ was used as a protein loading control. (C) Nuclear extracts from SMMC-7721, NC, MR1-C6 and MR1-D4 cells were detected by western blotting to assess the levels of nuclear $\beta$-catenin. Lamin B1 served as the nuclear loading control. (D) NC, MR1-C6 and MR1-D4 cells were transiently transfected with TOPflash and FOPflash plasmids. Relative luciferase activity was calculated as the ratio of TOPflash/FOPflash luciferase activity, and each value was normalized to the luciferase activity of the internal control pRL-TK reporter plasmid. (E) mRNA levels of cyclin D1 and c-Myc in NC, MR1-C6 and MR1-D4 cells were detected by qRT-PCR and normalized to $\beta$-actin. Bars represent the relative mRNA level when compared to the NC cells. (F) Cell lysates were analyzed by western blotting for the expression of cyclin D1 and c-Myc. $\beta$-actin was used as a loading control. Data are expressed as the means \pm SDs of 3 independent experiments. "P $<0.05$ compared with NC.

A

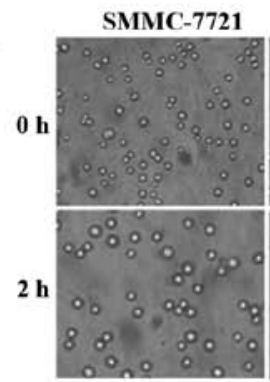

NC

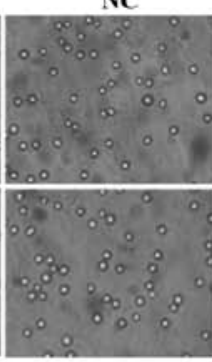

MR1-C6

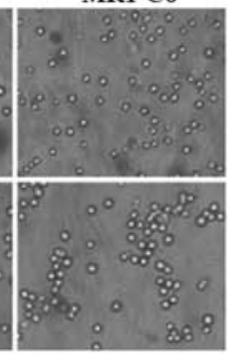

MR1-D4

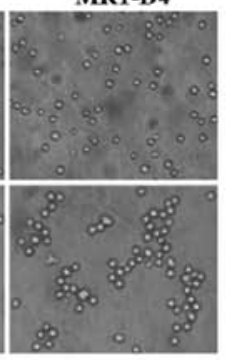

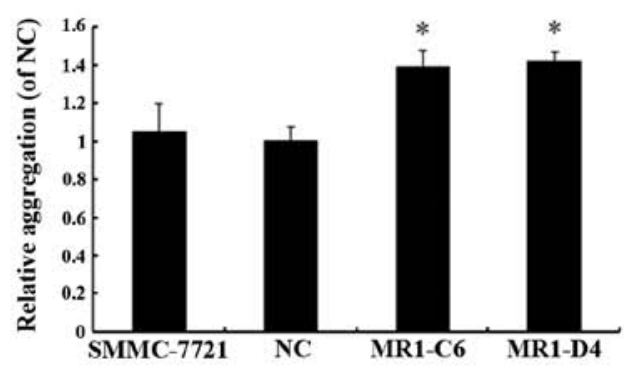

B
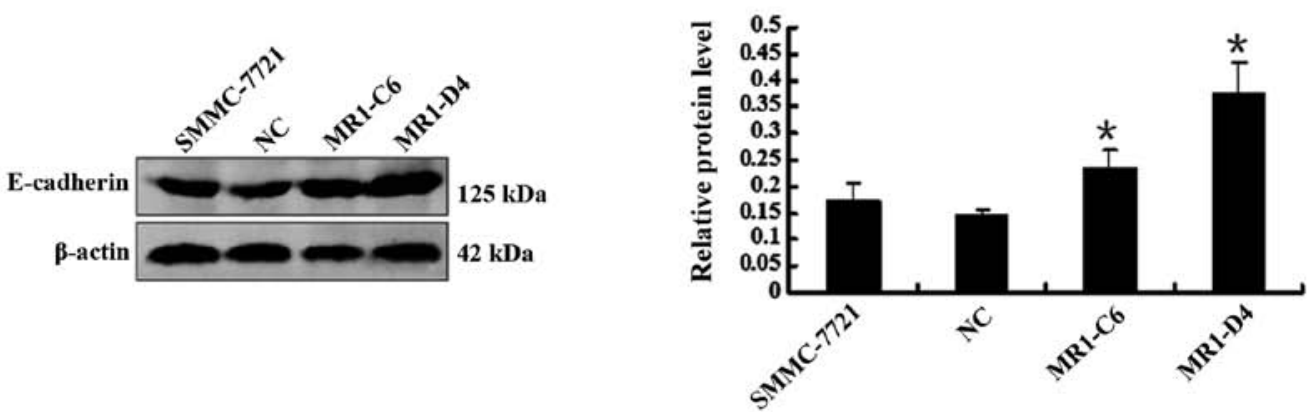

Figure 4. Knockdown of MUC1 expression enhances cell-cell aggregation. (A) Cell-cell aggregation assay; SMMC-7721, NC, MR1-C6 and MR1-D4 cells were seeded into 24 -well plates. Constant stirring method was performed to evaluate the cell-cell aggregation. The cell-cell aggregation index $=1$-(number of isolated cells at $2 \mathrm{~h} /$ number of isolated cells at $0 \mathrm{~h}$ ) and was normalized to the data obtained with the negative control ( $\mathrm{NC}$ ). $\mathrm{P}<0.05 \mathrm{compared}$ with $\mathrm{NC}$. (B) E-cadherin expression was detected by western blotting, and $\beta$-actin was used as a protein loading control. The bar charts represent relative protein levels calculated by the ratio of E-cadherin/ $\beta$-actin. Data are expressed as the means \pm SDs of 3 independent experiments. ${ }^{*}<<0.05$ compared with the $\mathrm{NC}$. 

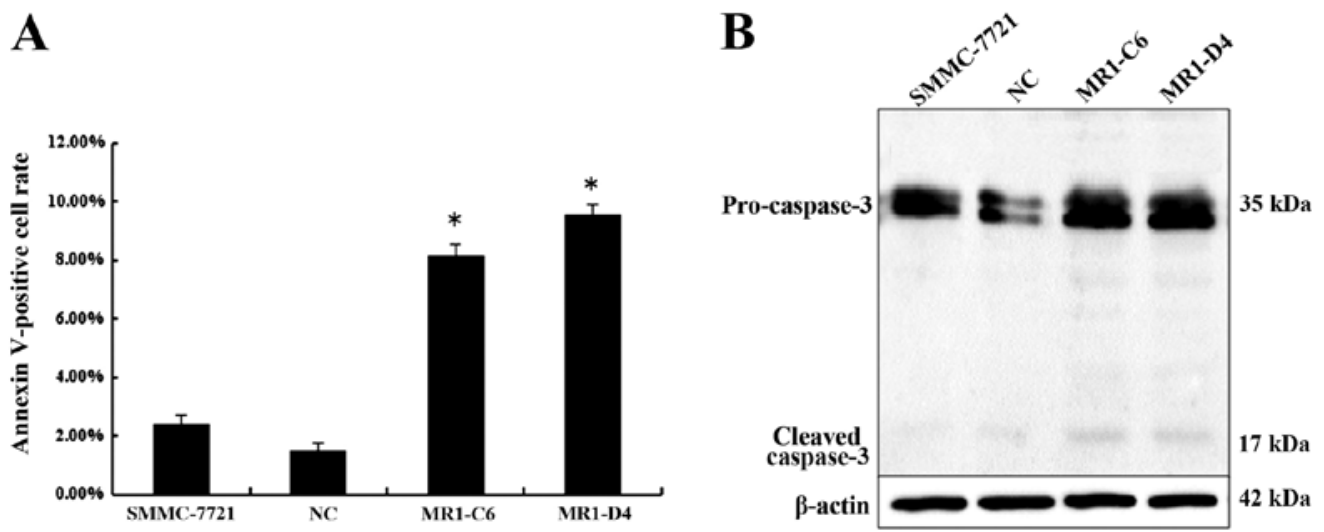

Figure 5. Knockdown of MUC1 expression induces apoptosis. (A) Cell apoptosis was determined by Annexin V-phycoerythrin (PE) staining. Annexin V-positive cell rate was calculated as (Annexin V-positive cell number / total cell number) x100\%. Data are expressed as the means \pm SDs of 3 independent experiments. ${ }^{*} \mathrm{P}<0.05$ compared with the NC. (B) Caspase- 3 expression was detected by western blotting and normalized to $\beta$-actin.
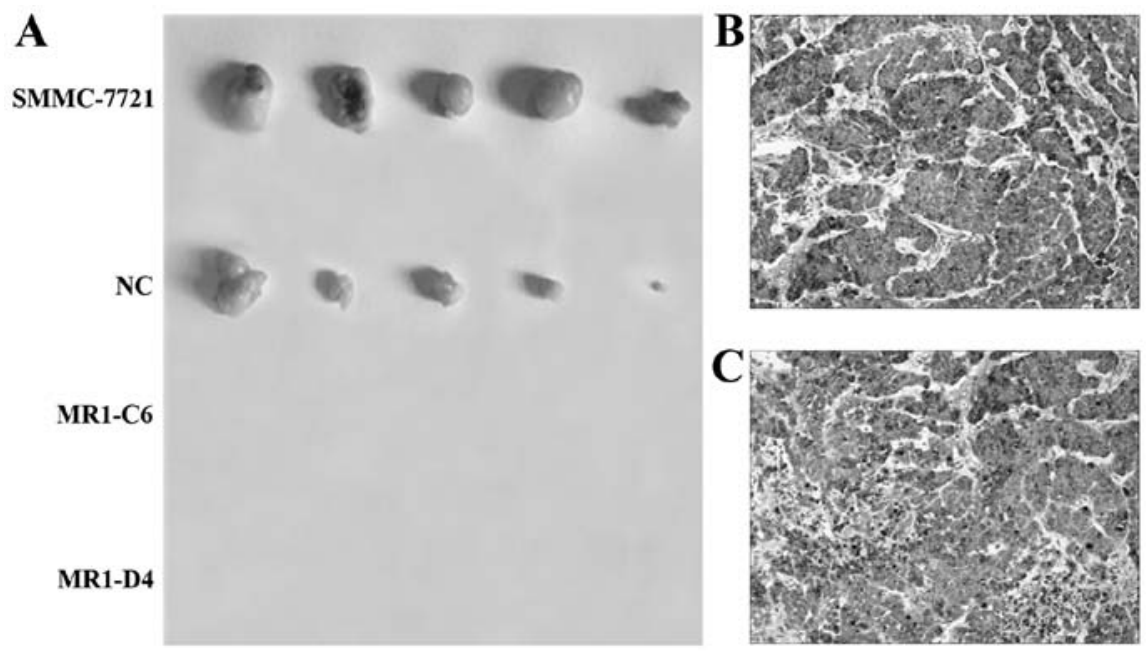

Figure 6. Knockdown of MUC1 expression suppresses tumor growth in vivo. (A) SMMC-7721, NC, MR1-C6 and MR1-D4 cells (2x106 cells) were subcutaneously injected into BALB/c nude mice to form subcutaneous tumors. On day 35 post-injection, tumors were dissected and photographed. Representative images were captured showing tumor sizes for each group. (B and C) Immunohistochemical staining for MUC1 in tumors from mice inoculated with SMMC-7721 cells and NC cells. Sections were examined on an inverted fluorescence microscope (IX71; Olympus) using a 100X objective.

cells. We developed a cell aggregation assay and examined the expression of E-cadherin, a molecular chaperone of $\beta$-catenin that plays an important role in cell aggregation. The results showed that the level of cell-cell aggregation and E-cadherin expression in the MR1-C6 and MR1-D4 cells were significantly enhanced when compared to $\mathrm{NC}$ or SMMC-7721 cells (Fig. 4A and $\mathrm{B})(\mathrm{P}<0.05)$, indicating that knockdown of MUC1 may enhance cell-cell aggregation by promoting E-cadherin expression. In addition, we performed scratch test migration and Matrigel invasion assays and found no significant differences in migration or invasion between MR1-C6 or MR1-D4 cells and NC or SMMC-7721 cells (data not shown).

Knockdown of MUCl expression induces apoptosis in SMMC-7721 cells. Next, we investigated the effect of MUC1 knockdown on cell apoptosis by Annexin V-phycoerythrin staining analysis. The results showed that more MR1-C6 and MR1-D4 cells were stained when compared with NC or SMMC-7721 cells. The percentage of Annexin V-positive cell population is plotted in Fig. 5A. Western blotting showed that caspase-3 cleavage was observed in MR1-C6 and MR1-D4 cells, while cleavage was not present in NC or SMMC-7721 cells (Fig. 5B). Taken together, these results indicate that knockdown of MUC1 induces apoptosis in SMMC-7721 cells.

Knockdown of MUCl expression suppresses tumor growth in vivo. To evaluate the effects of MUC1 expression on tumorigenesis in vivo, SMMC-7721, NC, MR1-C6 and MR1-D4 cells were inoculated subcutaneously into BALB/c nude mice to establish a subcutaneous transplant tumor model. Thirty-five days after tumor implantation, mice were sacrificed, and the tumors were dissected. As shown in Fig. 6A, tumor growth was observed in both the $\mathrm{NC}$ and SMMC-7721 groups but not in the MR1-C6 and MR1-D4 groups. Immunohistochemical staining showed that MUC1 was significantly expressed by tumors from mice inoculated with SMMC-7721 and NC cells (Fig. 6B and C). These results indicate that knockdown of MUC1 significantly suppresses SMMC-7721 tumor growth in vivo. 
Table I. Gene expression microarray results by comparison between MR1-C6 or MR1-D4 and NC clone, by order of magnitude.

Genes downregulated >2-fold in MUC1-knockdown clones

Wnt/ $\beta$-catenin signal

Cyclin D1

C-myc

Transcription factor 3 (TCF3)

TEA domain family member1 (TEF1/TCF13)

Transcription factor 7-like 1 (TCF7L1)

$\mathrm{NF}-\kappa \mathrm{B}$ signal

Nuclear factor of $\kappa$ light polypeptide gene enhancer in

B-cells 1 (p105) (NF- $\kappa \mathrm{B} 1)$

Nuclear factor of $\kappa$ light polypeptide gene enhancer in

B-cells 2 (p49/p100) (NF-кB2)

Inhibitor of $\kappa$ light polypeptide gene enhancer in B-cells, kinase beta (I $\kappa \mathrm{BKB})$

Dystrophin-associated glycoprotein (DAG1)

Insulin signal

Insulin-like growth factor 2 receptor (IGF2R)

Insulin receptor substrate 2 (IRS2)

Insulin-like growth factor binding protein 7 (IGFBP7)

Eukaryotic translation initiation factor $4 \mathrm{E}$ binding protein 1 (4EBP1)

Inositol polyphosphate phosphatase-like 1 (SHIP2)

Ras homolog enriched in brain (Rheb)

TGF- $\beta$ signal

SMAD, mothers against DPP homolog $2(\operatorname{Smad} 2)$

SMAD, mothers against DPP homolog 3 (Smad3)

Cell division cycle 42 (GTP binding protein, $25 \mathrm{kDa}$ ) (CDC42)

Ras homolog gene family, member A (RhoA)

Basic helix-loop-helix domain containing, class B, 2 (bHLHB2)

MAPK signal

Mitogen-activated protein kinase kinase 5 (MAP2K5)

Mitogen-activated protein kinase kinase 6 (MAP2K6)

Growth factor receptor-bound protein 10 (GRB10)

RAB31, member RAS oncogene family (RAB31)

VEGF signal

Vascular endothelial growth factor B (VEGFB)

Cell adhesion and aggregation associated

Laminin, $\gamma 1$ (formerly LAMB2) (LAMC1)

Genes upregulated >2-fold in MUC1-knockdown clones

Cell proliferation associated

Glycoprotein hormones, $\alpha$ polypeptide (CGA)

Growth arrest and DNA-damage-inducible, $\gamma$ interacting protein 1 (GADD45GIP1)

Growth differentiation factor 2 (GDF2)

DnaJ (Hsp40) homolog, subfamily B, member 2 (DNAJB2)

SRY (sex determining region Y)-box 17 (SOX17)

Netrin 1 (NTN1)
Table I. Continued.

Genes upregulated >2-fold in MUC1-knockdown clones

Metallothionein 1E (MT1E)

Nuclear factor of $\kappa$ light polypeptide gene enhancer in B-cells inhibitor, $\beta$ (NFאBIB)

Activin A receptor type II-like 1 (ACVRL1)

ADAM metallopeptidase with thrombospondin

type I motif, 15 (ADAMTS15)

Cell adhesion and aggregation associated

Intercellular adhesion molecule 5 (ICAM5)

Tumor necrosis factor receptor superfamily, member 12A (CD266) (FN14)

CD36 molecule (thrombospondin receptor) (CD36) (FAT)

Clones MR1-C6, MR1-D4 and NC were analyzed by gene expression microarrays. Listed are genes with expression increased or decreased $>2$-fold in both MUC1-downregulated clones when compared to the control.

Global gene expression analysis in SMMC-7721 cells. To further explore the mechanisms of MUC1 gene silencing that lead to inhibited cell proliferation and enhanced aggregation of SMMC-7721 cells, we utilized gene expression microarrays to analyze differential gene expression in clones NC, MR1-C6 and MR1-D4. As shown in Table I, some genes related to proliferation and aggregation were differentially expressed between clones MR1-C6 or MR1-D4 and NC. A series of genes encoding molecules that related to proliferation in the Wnt/ $\beta$-catenin, NF- $\kappa \mathrm{B}, \mathrm{MAPK}$, insulin, TGF- $\beta$ and VEGF signaling pathways were expressed at significantly lower levels in the MUC1-knockdown clones MR1-C6 and MR1-D4. However, aggregation-related genes such as ICAM and FAT were expressed at significantly higher levels. These results indicate that knockdown of MUC1 influenced a number of signaling pathways related to cell proliferation and cell-cell aggregation in SMMC-7721 cells, affecting the occurrence and development of HCC.

\section{Discussion}

In the present study, we investigated the effects of MUC1 knockdown on the HCC cell line SMMC-7721. SMMC-7721MUC1-knockdown stable clones were generated by RNA interference. We identified three independent MUC1knockdown clones, MR1-C6, MR1-D4 and MR1-D9, and one negative control clone, NC, via immunofluorescence staining, PCR and western blotting. We selected the clones MR1-C6 and MR1-D4 due to their higher silencing efficiency and the $\mathrm{NC}$ clone to study in vitro cell proliferation, colony formation, cell cycle, migration, invasion, aggregation and cell apoptosis as well as to evaluate in vivo tumorigenesis.

We found that the MUC1-silenced clones MR1-C6 and MR1-D4 had significantly inhibited cell proliferation, similar to results described by Tsutsumida et al (38), who showed that knockdown of MUC1 in pancreatic carcinoma cells (S2-013) significantly decreased cell proliferation. Raina et al (39) 
showed that $3 \mathrm{Y} 1$ cells overexpressing MUC1 could induce malignant transformation and enhance colony formation. Thus, we also utilized the colony formation assay to detect the effect of MUC1 gene silencing in MR1-C6 and MR1-D4 cells, showing a significant reduction in colony formation, further confirming that knockdown of MUC1 negatively regulated HCC cell proliferation. Subsequently, we performed cell cycle analysis and found that knockdown of MUC1 induced cell cycle arrest in the S-phase. In addition, we observed that knockdown of MUC1 induced apoptosis in SMMC-7721 cells, which is consistent with previous studies (40-42). Moreover, tumor growth was not observed in mice injected with MR1-C6 and MR1-D4 cells. These results suggest that MUC1 plays an important role in HCC tumorigenesis.

Several published studies (43-45) have shown that MUC1-CT interacts with $\beta$-catenin to form a complex and regulate the cellular localization of $\beta$-catenin. Nuclear translocation of $\beta$-catenin can activate cyclin D1 and c-Myc expression and stimulate cell proliferation, thus contributing to tumorigenesis and tumor progression. Our current study showed that MUC1-CT could interact with $\beta$-catenin and that knockdown of MUC1 in SMMC-7721 cells increased cytoplasmic levels of $\beta$-catenin but decreased the nuclear translocation of $\beta$-catenin, reduced the activity of TCF, downregulated expression of cyclin D1 and c-Myc, and arrested the cell cycle in S-phase. These results provide a potential mechanistic explanation of how MUC1 knockdown decreased the proliferation of SMMC7721 cells in vitro.

To determine the effect of MUC1 on SMMC-7721 cell migration, invasion and aggregation, we conducted the scratch test, Matrigel invasion and aggregation assays, respectively. The results showed that knockdown of MUC1 promoted cell-cell aggregation when compared to controls. Cell-cell aggregation is mainly mediated by the cadherin complex, which can maintain epidermal morphogenesis of epidermal cells and is involved in cell-cell interactions. Yuan et al (46) showed that MUC1-silenced cells could increase the cell-cell aggregation of breast cancer cells and lead to higher expression of E-cadherin. Our result showed that E-cadherin expression was significantly increased in MR1-C6 and MR1-D4 cells, indicating that knockdown of MUC1 may enhance cell-cell aggregation by promoting E-cadherin expression. In addition, we found no significant differences in cell migration or invasion activity between MUC1-silenced clones and controls, which is consistent with Costa et al (37). The reasons why MUC1 gene silencing did not affect HCC cell migration and invasion require further study.

To explore the mechanisms of MUC1 gene silencing that lead to inhibited cell proliferation and enhanced aggregation of SMMC-7721 cells, we further utilized microarrays to measure global gene expression and analyze differential gene expression between MUC1-silenced MR1-C6 or MR1-D4 cells and the NC cells. We showed that a series of genes encoding molecules in the $\mathrm{Wnt} / \beta$-catenin, NF- $\kappa \mathrm{B}, \mathrm{MAPK}$, insulin, VEGF and TGF- $\beta$ signaling pathways were influenced by knockdown of MUC1, and these may contribute to the phenotypic alterations observed. However, the mechanisms underlying how MUC1 regulates TGF- $\beta$ signaling pathway remain unclear and related experiments are being carried out.
In conclusion, our study shows for the first time that MUC1 expression influences the proliferation, apoptosis and cell-cell aggregation of HCC cell line SMMC-7721. These results indicate that MUC1 plays an important role in HCC tumorigenesis, and further suggest that MUC1 may be a potential target for HCC treatment.

\section{Acknowledgements}

This study was supported by grants from the China National Natural Science Foundation (No. 31170875) and the Major Development Programs for New Drugs of the Chinese Academy of Sciences during the 12th Five-Year Plan Period (No. 2011ZX09102- 001-36).

\section{References}

1. Kufe DW: Mucins in cancer: function, prognosis and therapy. Nat Rev Cancer 9: 874-885, 2009.

2. Duffy MJ: CA 15-3 and related mucins as circulating markers in breast cancer. Ann Clin Biochem 36 (Pt 5): 579-586, 1999.

3. Apostolopoulos V, Pietersz GA, Tsibanis A, et al: Pilot phase III immunotherapy study in early-stage breast cancer patients using oxidized mannan-MUC1 [ISRCTN71711835]. Breast Cancer Res 8: R27, 2006

4. Sugiura D, Aida S, Denda-Nagai K, et al: Differential effector mechanisms induced by vaccination with MUC1 DNA in the rejection of colon carcinoma growth at orthotopic sites and metastases. Cancer Sci 99: 2477-2484, 2008.

5. Zhang S, Zhang HS, Cordon-Cardo C, Ragupathi G and Livingston PO: Selection of tumor antigens as targets for immune attack using immunohistochemistry: protein antigens. Clin Cancer Res 4: 2669-2676, 1998.

6. Gendler S, Taylor-Papadimitriou J, Duhig T, Rothbard J and Burchell J: A highly immunogenic region of a human polymorphic epithelial mucin expressed by carcinomas is made up of tandem repeats. J Biol Chem 263: 12820-12823, 1988.

7. Ligtenberg MJ, Kruijshaar L, Buijs F, van Meijer M, Litvinov SV and Hilkens J: Cell-associated episialin is a complex containing two proteins derived from a common precursor. J Biol Chem 267: 6171-6177, 1992.

8. Kufe DW: Targeting the human MUC1 oncoprotein: a tale of two proteins. Cancer Biol Ther 7: 81-84, 2008.

9. Singh PK and Hollingsworth MA: Cell surface-associated mucins in signal transduction. Trends Cell Biol 16: 467-476, 2006.

10. Carraway KL III, Funes M, Workman HC and Sweeney C: Contribution of membrane mucins to tumor progression through modulation of cellular growth signaling pathways. Curr Top Dev Biol 78: 1-22, 2007.

11. Yu LG, Andrews N, Zhao Q, et al: Galectin-3 interaction with Thomsen-Friedenreich disaccharide on cancer-associated MUC1 causes increased cancer cell endothelial adhesion. J Biol Chem 282: 773-781, 2007.

12. Rahn JJ, Shen Q, Mah BK and Hugh JC: MUC1 initiates a calcium signal after ligation by intercellular adhesion molecule-1. J Biol Chem 279: 29386-29390, 2004.

13. Rahn JJ, Chow JW, Horne GJ, et al: MUC1 mediates transendothelial migration in vitro by ligating endothelial cell ICAM-1. Clin Exp Metastasis 22: 475-483, 2005.

14. Shen Q, Rahn JJ, Zhang J, et al: MUC1 initiates Src-CrkLRac1/Cdc42-mediated actin cytoskeletal protrusive motility after ligating intercellular adhesion molecule-1. Mol Cancer Res 6: 555-567, 2008.

15. Zhao Q, Barclay M, Hilkens J, et al: Interaction between circulating galectin-3 and cancer-associated MUC1 enhances tumour cell homotypic aggregation and prevents anoikis. Mol Cancer 9: $154,2010$.

16. Huang L, Chen D, Liu D, Yin L, Kharbanda S and Kufe D: MUC1 oncoprotein blocks glycogen synthase kinase 3beta-mediated phosphorylation and degradation of beta-catenin. Cancer Res 65: 10413-10422, 2005.

17. Li Y, Kuwahara H, Ren J, Wen G and Kufe D: The c-Src tyrosine kinase regulates signaling of the human DF3/MUC1 carcinomaassociated antigen with GSK3 beta and beta-catenin. J Biol Chem 276: 6061-6064, 2001. 
18. Pandey $\mathrm{P}$, Kharbanda $\mathrm{S}$ and Kufe D: Association of the DF3/MUC1 breast cancer antigen with Grb2 and the Sos/Ras exchange protein. Cancer Res 55: 4000-4003, 1995.

19. Wei X, Xu H and Kufe D: Human mucin 1 oncoprotein represses transcription of the p53 tumor suppressor gene. Cancer Res 67 1853-1858, 2007.

20. Singh PK, Behrens ME, Eggers JP, et al: Phosphorylation of MUC1 by Met modulates interaction with p53 and MMP1 expression. J Biol Chem 283: 26985-26995, 2008.

21. Schroeder JA, Thompson MC, Gardner MM and Gendler SJ: Transgenic MUC1 interacts with epidermal growth factor receptor and correlates with mitogen-activated protein kinase activation in the mouse mammary gland. J Biol Chem 276 13057-13064, 2001.

22. Lau SK, Shields DJ, Murphy EA, et al: EGFR-mediated carcinoma cell metastasis mediated by integrin alphavbeta5 depends on activation of $\mathrm{c}-\mathrm{Src}$ and cleavage of MUC1. PLoS One 7: e36753, 2012.

23. Ahmad R, Raina D, Trivedi V, et al: MUC1 oncoprotein activates the IkappaB kinase beta complex and constitutive NF-kappaB signalling. Nat Cell Biol 9: 1419-1427, 2007.

24. Ahmad R, Raina D, Joshi MD, et al: MUC1-C oncoprotein functions as a direct activator of the nuclear factor-kappaB p65 transcription factor. Cancer Res 69: 7013-7021, 2009.

25. Hu XF, Yang E, Li J and Xing PX: MUC1 cytoplasmic tail: a potential therapeutic target for ovarian carcinoma. Expert Rev Anticancer Ther 6: 1261-1271, 2006.

26. Kufe DW: Functional targeting of the MUC1 oncogene in human cancers. Cancer Biol Ther 8: 1197-1203, 2009.

27. Klinge CM, Radde BN, Imbert-Fernandez Y, et al: Targeting the intracellular MUC1 C-terminal domain inhibits proliferation and estrogen receptor transcriptional activity in lung adenocarcinoma cells. Mol Cancer Ther 10: 2062-2071, 2011.

28. Hattrup CL, Bradley JM, Kotlarczyk KL, et al: The MUC1 cytoplasmic tail and tandem repeat domains contribute to mammary oncogenesis in FVB mice. Breast Cancer (Auckl) 1: $57-63,2008$

29. Wang L, Ma J, Liu F, et al: Expression of MUC1 in primary and metastatic human epithelial ovarian cancer and its therapeutic significance. Gynecol Oncol 105: 695-702, 2007.

30. Kohlgraf KG, Gawron AJ, Higashi M, et al: Contribution of the MUC1 tandem repeat and cytoplasmic tail to invasive and metastatic properties of a pancreatic cancer cell line. Cancer Res 63: 5011-5020, 2003.

31. Inagaki Y, Tang W, Xu H, et al: Sustained aberrant localization of KL-6 mucin and beta-catenin at the invasion front of human gastric cancer cells. Anticancer Res 31: 535-542, 2011.

32. Bozkaya G, Korhan P, Cokakli M, et al: Cooperative interaction of MUC1 with the HGF/c-Met pathway during hepatocarcinogenesis. Mol Cancer 11: 64, 2012.

33. Yuan SF, Li KZ, Wang L, et al: Expression of MUC1 and its significance in hepatocellular and cholangiocarcinoma tissue. World J Gastroenterol 11: 4661-4666, 2005.
34. Livak KJ and Schmittgen TD: Analysis of relative gene expression data using real-time quantitative PCR and the 2(-Delta Delta C(T)) method. Methods 25: 402-408, 2001

35. Veeravalli KK, Chetty C, Ponnala S, et al: MMP-9, uPAR and cathepsin B silencing downregulate integrins in human glioma xenograft cells in vitro and in vivo in nude mice. PLoS One 5 : e11583, 2010

36. Wang F, Li Q, Ni W, et al: Expression of human full-length MUC1 inhibits the proliferation and migration of a B16 mouse melanoma cell line. Oncol Rep 30: 260-268, 2013.

37. Costa NR, Paulo P, Caffrey T, Hollingsworth MA and Santos-Silva F: Impact of MUC1 mucin downregulation in the phenotypic characteristics of MKN45 gastric carcinoma cell line. PLoS One 6: e26970, 2011.

38. Tsutsumida H, Swanson BJ, Singh PK, et al: RNA interference suppression of MUC1 reduces the growth rate and metastatic phenotype of human pancreatic cancer cells. Clin Cancer Res 12: 2976-2987, 2006

39. Raina D, Kharbanda S and Kufe D: The MUC1 oncoprotein activates the anti-apoptotic phosphoinositide 3-kinase/Akt and Bcl-xL pathways in rat 3Y1 fibroblasts. J Biol Chem 279: 20607-20612, 2004.

40. Raina D, Ahmad R, Chen D, Kumar S, Kharbanda S and Kufe D: MUC1 oncoprotein suppresses activation of the ARF-MDM2-p53 pathway. Cancer Biol Ther 7: 1959-1967, 2008.

41. Agata N, Ahmad R, Kawano T, Raina D, Kharbanda S and Kufe D: MUC1 oncoprotein blocks death receptor-mediated apoptosis by inhibiting recruitment of caspase-8. Cancer Res 68 : 6136-6144, 2008

42. Ahmad R, Alam M, Rajabi H and Kufe D: The MUC1-C oncoprotein binds to the $\mathrm{BH} 3$ domain of the pro-apoptotic $\mathrm{BAX}$ protein and blocks BAX function. J Biol Chem 287: 2086620875, 2012

43. Li Y, Liu D, Chen D, Kharbanda S and Kufe D: Human DF3/MUC1 carcinoma-associated protein functions as an oncogene. Oncogene 22: 6107-6110, 2003.

44. Wen Y, Caffrey TC, Wheelock MJ, Johnson KR and Hollingsworth MA: Nuclear association of the cytoplasmic tail of MUC1 and beta-catenin. J Biol Chem 278: 38029-38039, 2003.

45. Li Y, Yi H, Yao Y, et al: The cytoplasmic domain of MUC1 induces hyperplasia in the mammary gland and correlates with nuclear accumulation of beta-catenin. PLoS One 6: e19102, 2011.

46. Yuan Z, Wong S, Borrelli A and Chung MA: Downregulation of MUC1 in cancer cells inhibits cell migration by promoting E-cadherin/catenin complex formation. Biochem Biophys Res Commun 362: 740-746, 2007. 